\title{
Subsequent reactions were common and often more serious than the initial reactions of children with peanut allergy
}

\author{
Vander Leek TK, Liu AH, Stefanski K, et al. The natural history of peanut allergy in young children and its association \\ with serum peanut-specific IgE. J Pediatr 2000 Dec;137:749-55.
}

QUESTION: In young children with peanut allergy diagnosed before 4 years of age,
what is the nature and rate of adverse reactions caused by accidental peanut exposure?

Design

Cohort study with a median of 5.9 years (range 1.4 to 22.4 y) of follow up.

Source of funding: no external funding.

For correspondence: Dr S A Bock,

Department of

Pediatrics, National

Jewish Medical and

Research Center, 1400

Jackson Street, Room

J211, Denver, $C O$

80206, USA. Fax +1

3034435030 .

A modified version of this abstract appears in

Evidence-Based

Nursing.

\section{Setting \\ Boulder, Colorado, USA.}

\section{Patients}

102 children with clinical peanut hypersensitivity diagnosed before their fourth birthday. Children were included if they had a convincing history of clinical peanut hypersensitivity; a positive double blind or a placebo controlled food challenge response to peanuts, or both; and a positive skin prick test response to peanuts. Data for 83 children (81\%) (median age 2.4 y, 69\% boys) were available for the analysis.

\section{COMMENTARY}

The incidence of peanut allergy, which commonly occurs in the US and in the UK, appears to be increasing, possibly because of earlier exposure to peanut antigen in the diet. Reactions can be life threatening (eg, laryngeal oedema or asphyxia), and sensitivity is less likely to be lost with advancing age than is the case with milk or egg allergy. Unfortunately, peanut protein avoidance, the key to management, is problematic given the widespread inclusion of peanut in different foods. Patients and their caregivers therefore are often given an injection device for self administration of epinephrine (adrenaline) for emergency use.

This study included children with well defined reactions to peanut in an attempt to define the frequency and type of adverse event precipitated by subsequent accidental exposure. Adverse reactions were relatively common, which emphasises the difficulty of antigen avoidance. Among those children who had mild reactions on first exposure, almost half had life threatening symptoms on later accidental exposure. Those children who had only skin symptoms during first and subsequent peanut exposure had lower concentrations of serum peanut specific IgE than those who had respiratory or gastrointestinal symptoms. The overlap between groups, however, meant that $\operatorname{IgE}$ measurement could not accurately predict later reaction. Low IgE concentrations were found in 4 patients who subsequently lost hypersensitivity to peanut, an observation that may serve as a guide to identifying those children who should later have formal challenges done.

This study shows that, despite dietary counselling, accidental peanut exposure is common. Furthermore, initial mild reactions in some children do not ensure freedom from more severe symptoms provoked by subsequent accidental exposure. Therefore, extending the use of self administered adrenaline devices to these patients rather than limiting their prescription to children with initial life threatening symptoms might be necessary. Clinicians, however, should consider the findings by Sicherer $e t$ al that many doctors are unfamiliar with the practicalities of the adrenaline devices they prescribe, and as a result, they frequently fail to effectively teach families and children how to administer this potentially life saving treatment.

John Puntis, BM, DM General Infirmary at Leeds, Leeds, UK

1 Sicherer SH, Forman JA, Noone SA. Use assessment of self-administered epinephrine among food-allergic children and pediatricians. Pediatrics 2000;105:359-62.

\section{Assessment of prognostic factors}

Initial assessment records: severity of symptoms (nonlife threatening or potentially life threatening); organ system involvement (skin, respiratory, gastrointestinal, or other); and serum peanut specific immunoglobulin E (IgE) concentrations (in 51 of 83 [61\%] children).

\section{Main outcome measures}

Subsequent adverse reactions after a first peanut exposure were assessed at least yearly.

\section{Main results}

50 children (60\%) had a total of 115 adverse reactions caused by accidental peanut exposure during follow up (mean 0.33 adverse reactions/y). During their first reaction, 12 children (14\%) had skin symptoms alone after only skin contact with peanuts; 26 (31\%) had skin symptoms alone after eating peanuts; and 45 (54\%) had respiratory symptoms, gastrointestinal symptoms, skin symptoms, or all 3 .

61 children $(73 \%)$ had non-life threatening first reactions to accidental peanut exposure, and $22(27 \%)$ had potentially life threatening first reactions. Of the 61 children with non-life threatening first reactions, 43 had a subsequent reaction, with 19 (44\%) being potentially life threatening. Of the 22 children with potentially life threatening first reactions, 17 had a subsequent reaction, with $12(71 \%)$ being potentially life threatening. Overall, of the 60 children $(72 \%)$ with $\geqslant 1$ subsequent reaction, $31(52 \%)$ had $\geqslant 1$ potentially life threatening subsequent reaction.

Of the 51 children who had their serum peanut specific IgE concentrations measured, those who had subsequent skin symptoms alone $(n=11)$ had a lower median serum peanut specific $\operatorname{IgE}$ concentration than those who had subsequent respiratory symptoms or gastrointestinal symptoms, or both $(\mathrm{n}=40)(1.25 v 11.65$ $\left.\mathrm{kU}_{\mathrm{A}} / \mathrm{l}, \mathrm{p}=0.004\right)$. However, no threshold level for serum peanut specific IgE existed below which only skin symptoms occurred.

\section{Conclusion}

Most young children with clinical peanut hypersensitivity continued to have adverse reactions to accidental peanut exposure; however, symptoms experienced during a subsequent adverse peanut reaction were not easily predictable either from symptoms experienced during a first reaction or from immunoglobulin $\mathrm{E}$ concentrations. 